\title{
Construction of predictive models of spatial resolution and signal to noise ratio (SNR) based on modification of the FOV in Magnetic Resonance - a quantitative approach to a daily event
}

\begin{abstract}
Obtain optimal image quality in MRI is a daily challenge, which is why the management and understanding of the acquisition parameters and their interactions is paramount. The objective of this research is to build predictive models of three image quality factors (FOV, SNR and SR) for two coils common use and high load (Brain and Knee). The result was to obtain quantitative models to predict changes in image resolution (RE) and Signal to Noise Ratio (SNR) against possible changes in the dimensions of the FOV in image acquisition. It has also been able to establish an accurate and objective communication between the Radiologist and Medical Technologist, it has been established to track the quality of the coils studied and has established a standardization of these quality factors among all technical staff.
\end{abstract}

Keywords: image resolution, radiologist, MRI, brain, knee
Volume 9 Issue 2 - 2018

\author{
Gonzalo Araya Rojas, Claudio Silva Fuente, \\ Alba \\ Departamento de Imágenes Clínica Alemana Santiago de Chile, \\ Chile \\ Correspondence: Gonzalo Araya Rojas, Departamento de \\ Imágenes Clínica Alemana Santiago de Chile, Chile, \\ Email gonzalorayos@gmail.com
}

Received: February 21, 2018 | Published: March 27, 2018

\section{Introduction}

Daily imaging Magnetic Resonance Imaging (MRI) ${ }^{1}$ is always a challenge as obtaining adequate diagnostic image quality requires technicians to constantly adjust each patient's unique characteristics studied factors. Radiologist demands optimal image quality, and to achieve this there must be effective communication, including technical terms of image quality between the Medical Technologist and Radiologist. Today, this technique communication between the Radiologist and Medical Technologist is scarce and where there is little objective framed in terms that come to define unclear and nonquantifiable improvement plans.

Factors most frequently used image quality they are mainly the field of view (FOV), Signal to Noise Ratio (SNR) and spatial resolution (SR). The objective of this research is to build predictive models of three factors image quality (FOV, SNR and SR) for two coils frequent use and high loading usage (Brain and knee) in 2 MRI equipment (Skyra Siemens 3T and GE Signa 1.5T) in standard phantoms. This will allow objectively and with high accuracy values, the value added will know what the impact of SNR and SR against changes in the acquisition FOV exam. This produces a more accurate, objective and reproducible technical communication in time to changes in acquisition protocols will be achieved, in addition to increased local quality control of our MRI equipment and standardization of these factors with all Medical Technologists manipulate the resonator in which the models were built. ${ }^{2}$

\section{Material and methods}

Postprocessing software images Image J, Microsoft Excell. Images were acquired with phantom team Skyra Siemens 3T GE Signa 1.5T Equipment with two coils; brain 32 channels and 16 channels knee, where a scaling increased FOV are simulated. These images were analyzed with ImageJ software, determining the spatial resolution and signal noise for each of the images, using the methodology of the Spanish Quality Assurance in Diagnostic Radiology 2011 revision. Graphing models FOV v/s RE and FOV v/s RSR were built. A linear fit is performed to approximate the dependence between variables, the equations obtained for each case.

\section{Results and discussion}

Then disclosed patterns resulting from the analysis of images obtained for equipment with Siemens 3T Skyra brain coil 32 channels. With the quantitative relationship obtained we can objectively predict changes in image resolution against possible changes in the dimensions of the FOV in the acquisition of the image, thus we can anticipate whether the changes made in the FOV will be suitable for the expected quality by radiologists (Figure 1).

$$
y=0,0041 x-0,6348
$$

As an example we can say that with a FOV of $300 \mathrm{~mm}$, we would obtain a resolution as reflected voxel size of $0.6 \mathrm{~mm}$ (Figure 2).

$$
\begin{aligned}
& y=0,0041 x-0,6348 \\
& y=0,0041(300)-0,6348 \\
& y=0,5952 \mathrm{~mm}
\end{aligned}
$$

Usually these calculations are performed by the computer software of $\mathrm{MRI}^{3}$ to show the user, but the added value that has the power to build these models with this methodology is to have a local quality control for each team and for each coil use our center, thus we maintain a leading role in the performance of our teams. Constructing these models for each team and to maintain coil allows immediate consultation tool to predict the image quality you get from potential changes in these factors acquisition. As future research is to make these models in all remaining acquisition parameters and their interactions with each other. ${ }^{4,5}$ 


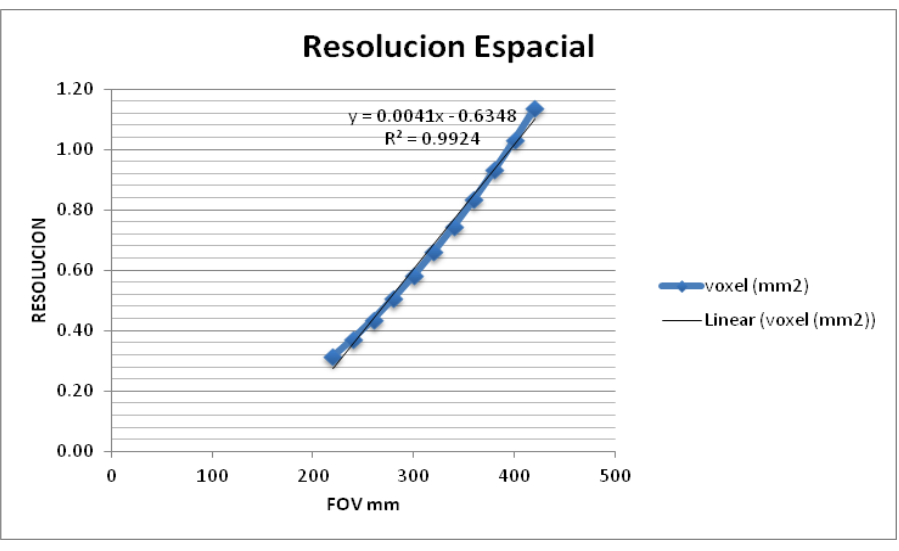

Figure I FOV v/s resolution linear fit with the quantitative model.

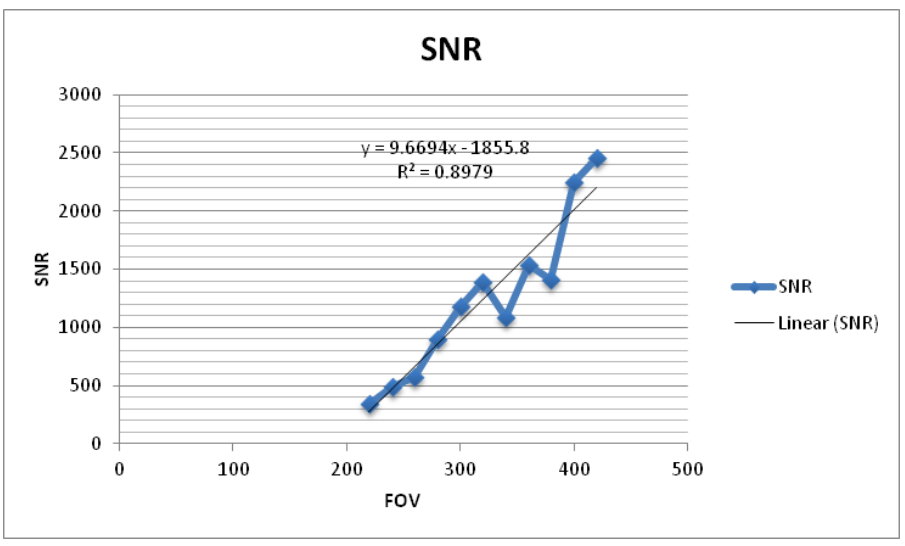

picture quality if you propose a change in the FOV of the image to be acquired. It has succeeded in establishing an accurate and objective communication between the Radiologist and Medical Technologist with quantitative, reliable and reproducible over time in the quality factors studied in this research measures. It has managed to establish monitoring and quality control for the coils studied, being sensitive to minor changes in the quality of acquisition of the resonance signal.

It has established a standardization of these quality factors among all the Staff of Medical Technologists of our institution.

\section{Acknowledgements}

None.

\section{Conflict of interest}

The author declares there is no conflict of interest.

\section{References}

1. Spanish Protocol Quality Assurance in Diagnostic Radiology. Magnetic Resonance Imaging Equipment. 2011;275-285.

2. Acceptance Testing and Quality Assurance Procedures for Magnetic Resonance Imaging Facilities. USA: Medical Physics Publishing; 2010.

3. Magnetic resonance imaging quality manual control (2004). USA: MRI Committee on Accreditation; 2006.

4. ACR Technical Standard for Diagnostic Medical Physics Performance Monitoring of Magnetic Resonance Imaging. USA: MRI Equipment; 2009.

5. Dobins JT III, Samei E, Ranger NT, et al. Intercomparison of methods for image quality characterization. II. Noise Power Spectrum. Med Phys. 2006;33(5):1465-1475.

Figure $2 \mathrm{FOV} v / \mathrm{s}$ SNR and linear fit with the quantitative model.

\section{Conclusion}

It has managed to build predictive models for SNR and SR against the FOV changes in the acquisition of magnetic resonance imaging. These models allow to know a priori what the impact of these 2 factors 